\title{
Desempenho, características de carcaça e qualidade de carne de frangos de corte suplementados com selênio orgânico ${ }^{1}$
}

\section{Performance, broiler carcass and meat quality characteristics, supplemented with organic selenium}

\author{
Lara Gonçalves de Medeiros²; Alexandre $\mathrm{Oba}^{3 *}$; Massami Shimokomaki \\ João Waine Pinheiro ${ }^{3}$; Caio Abércio da Silva ${ }^{3}$; Adriana Lourenço Soares ${ }^{5}$; \\ Aniele Pissinati²; Maurício de Almeida ${ }^{2}$
}

\section{Resumo}

\begin{abstract}
O trabalho teve como objetivo avaliar as características produtivas, de rendimento e qualidade de carne de frangos de corte alimentados com dietas suplementadas com diferentes níveis de selênio orgânico ( 0 ; 0,2; 0,4 e 0,6 mg Se/kg de ração). Foram utilizados 300 pintainhos de corte machos, da linhagem Cobb, com um dia de idade. Foi adotado um delineamento inteiramente ao acaso, com quatro tratamentos (níveis de selênio orgânico), com cinco repetições de 15 aves por parcela experimental. Foram avaliados o desempenho, rendimento de carcaça e cortes e a qualidade de carne de frangos de corte. Os resultados de desempenho mostram que a suplementação de selênio não alterou os dados produtivos, porém, reduziu linearmente a gordura abdominal da carcaça. Quanto à qualidade de carne, a suplementação de selênio orgânico aumentou linearmente o $\mathrm{pH}$ da carne do peito e reduziu a perda de água por pressão e a força de cisalhamento, melhorando assim a qualidade da carne das aves.
\end{abstract}

Palavras-chave: Aves, ganho de peso, microminerais, nutrição, $\mathrm{pH}$

\begin{abstract}
This research was aimed at assessing parameters such like productive and meat quality characteristics of broilers fed on diets with different levels of organic selenium $(0 ; 0,2 ; 0,4$ e $0 ; 6 \mathrm{mg} \mathrm{Se} / \mathrm{kg}$ of feed). Three hundred male one-day old chickens have been submitted to a completely randomized design, with five repetitions of 15 birds per experimental parcel. Characteristics such like performance, yield grade, cuts, and meat quality have been assessed. The results revealed that the supplementation with selenium did not affect productive characteristics of the broilers. However, it produced a linear reduction on the abdominal fat of the carcasses assessed. Regarding meat quality, the supplementation with organic selenium linearly increased the $\mathrm{pH}$ levels at the breast. Besides, it linearly reduced the loss of water by pressure and the shear force, which in turn improved the final quality of the meat.
\end{abstract}

Key words: Chickens, nutrition, $\mathrm{pH}$, trace element, weight gain

\footnotetext{
1 Parte da dissertação de Mestrado em Ciência Animal, do primeiro autor, Universidade Estadual de Londrina, UEL, Londrina, PR.

${ }^{2}$ Discente(s) de Pós-graduação em Ciência Animal, Universidade Estadual de Londrina, UEL, Londrina, PR. E-mail: laragmedeiros@hotmail.com; aniele_pissinati@hotmail.com; mauricio_alm_jn@hotmail.com

${ }^{3}$ Profs. Drs. do Dept ${ }^{\circ}$ de Zootecnia, UEL, Londrina, PR. E-mail: oba@uel.br; jwaine@uel.br; casilva@uel.br

${ }^{4}$ Prof. Dr. da Universidade Federal Tecnológica, UTFPR, Londrina, PR. E-mail: mshimo@uel.br

${ }^{5}$ Prof $^{\mathrm{a}}$. Dr ${ }^{\mathrm{a}}$. do Dept ${ }^{\mathrm{o}}$ de Ciência e Tecnologia de Alimentos, UEL, Londrina, PR. E-mail: adri.soares@uel.br

*Autor para correspondência
} 


\section{Introdução}

De uma forma geral, os microminerais possuem baixa taxa de absorção pelos animais, principalmente quando são fornecidos na forma inorgânica. A utilização dos chamados minerais orgânicos é uma alternativa interessante para maximizar a produção animal, já que tais minerais possuem maior biodisponibilidade, e atuam com maior eficiência no organismo (CLOSE, 1998).

O selênio é um micromineral essencial para funções orgânicas como crescimento, reprodução, prevenção de várias doenças e manutenção da integridade dos tecidos. Os efeitos da sua suplementação em dietas de aves podem variar de acordo com a fonte utilizada, seja ela orgânica ou inorgânica (CANTOR et al., 1997). Segundo Moreira et al. (2001), o selênio orgânico favorece as características produtivas em frangos de corte.

Outro aspecto importante desse mineral é a sua ação relacionada com a síntese de selenoaminoácidos e seleno-proteínas (MOREIRA et al., 2001). As seleno-proteínas desempenham importantes funções no metabolismo animal (EDENS; GOWDY; SEFTON, 2003). Entre estas, está a seleno-proteína iodotironina deiodinase que é responsável pela conversão da forma inativa da tiroxina (T4) para a forma ativa 3,5,3'triiodotironina (T3) (ARTHUR et al., 1990).

O hormônio T3 atua sobre o crescimento do animal através do turnover proteico (JIANHUA; OHTSUKA; HAYASHI, 2000). A deficiência de selênio poderia afetar este turnover e, consequentemente, o crescimento animal, pela redução da síntese de T3 (DAHLKE et al., 2005).

A deficiência de selênio é reconhecida como um importante fator que afeta a saúde humana, em todo mundo. Desta maneira, encontrar formas de disponibilidade deste mineral nos alimentos, bem como verificar como ele pode influenciar no rendimento de cortes e na qualidade da carne das aves torna-se um importante campo de pesquisa (VIARO; VIARO; FLECK, 2001).

Desta forma, o presente estudo teve como objetivo avaliar o efeito da suplementação dietética de diferentes níveis de selênio orgânico sobre os parâmetros de desempenho, rendimento de carcaça e da qualidade de carne em frangos de corte.

\section{Material e Métodos}

O experimento foi realizado no galpão experimental da Fazenda Escola e no Laboratório de Análise e Nutrição Animal (LANA) da Universidade Estadual de Londrina (UEL)- Paraná. Foram utilizados 300 pintainhos de corte machos da linhagem Cobb 700 com um dia de idade. As aves foram criadas de acordo com manejo comercial, com água e alimento ad libitum durante todo o período experimental de 42 dias, o qual foi dividido em quatro fases: pré-inicial ( $1-7$ dias de idade), inicial ( 8 - 21 dias de idade), crescimento $(22-35$ dias de idade) e terminação (36 - 42 dias de idade).

As rações experimentais (Tabela 1) atendiam as exigências mínimas preconizadas por Rostagno et al. (2011), sendo específicas para cada fase, inclusive para os níveis de selênio, fornecido juntamente com suplemento vitamínico-mineral utilizado na forma de selenito de sódio. Os tratamentos experimentais consistiam na suplementação de diferentes níveis de selênio orgânico $(0 ; 0,2 ; 0,4$ e $0,6 \mathrm{mg} \mathrm{Se} / \mathrm{kg}$ de ração). Adotou-se um delineamento inteiramente ao acaso, com quatro tratamentos (níveis de selênio orgânico), com cinco repetições de 15 aves por parcela experimental. Para as análises de carne, a unidade experimental era a ave, sendo utilizada 10 repetições por tratamento.

O selênio orgânico utilizado foi produzido através da liofilização de biomassa de levedura com selênio bioacumulado desenvolvido pelo departamento de Engenharia de Bioprocessos e Biotecnologia da Universidade Federal do Paraná. O produto foi produzido a partir da fermentação de melaço de cana $\left(7,5^{\circ}\right.$ Brix $)$ e extrato de levedura $(5 \mathrm{~g} / \mathrm{L})$, sendo que o microorganismo utilizado foi Candida pelliculosa BARU 05. A biomassa produzida possuía uma concentração de $599 \mathrm{mg} \mathrm{Se} /$ $\mathrm{kg}$ de biomassa seca. 
Tabela 1. Composição percentual e calculada das rações nas diferentes fases de criação de frangos de cortes criados até os 42 dias de idade.

\begin{tabular}{|c|c|c|c|c|}
\hline Ingredientes (\%) & Pré-Inicial & Inicial & Crescimento & Terminação \\
\hline Milho grão & 55,436 & 57,893 & 60,740 & 65,018 \\
\hline Soja farelo $45 \%$ & 38,013 & 35,151 & 31,580 & 27,683 \\
\hline Óleo de soja & 2,307 & 3,187 & 4,063 & 3,987 \\
\hline Fosfato bicálcico & 1,931 & 1,575 & 1,344 & 1,120 \\
\hline Calcário & 0,788 & 0,835 & 0,796 & 0,722 \\
\hline Sal comum & 0,507 & 0,482 & 0,457 & 0,445 \\
\hline Premix vit. mineral $^{1}$ & 0,400 & 0,400 & 0,400 & 0,400 \\
\hline L-Lisina $\mathrm{HCl}$ & 0,308 & 0,257 & 0,256 & 0,281 \\
\hline Dl-Metionina & 0,184 & 0,129 & 0,281 & 0,257 \\
\hline L-Treonina & 0,126 & 0,091 & 0,083 & 0,087 \\
\hline Total & 100 & 100 & 100 & 100 \\
\hline \multicolumn{5}{|l|}{ Calculada } \\
\hline Energia metabolizável (kcal/kg) & 2960 & 3050 & 3150 & 3200 \\
\hline Proteína bruta (\%) & 21,050 & 20,240 & 19,040 & 17,810 \\
\hline Metionina digestível (\%) & 0,519 & 0,449 & 0,408 & 0,376 \\
\hline Met. + cist. digestível (\%) & 0,994 & 0,817 & 0,734 & 0,676 \\
\hline Lisina digestível (\%) & 1,330 & 1,151 & 1,020 & 0,939 \\
\hline Treonina digestível (\%) & 0,865 & 0,748 & 0,663 & 0,610 \\
\hline Selênio (\%) & 0,0037 & 0,0033 & 0,0030 & 0,0023 \\
\hline Cálcio (\%) & 0,894 & 0,853 & 0,794 & 0,735 \\
\hline Fósforo disponível (\%) & 0,450 & 0,428 & 0,398 & 0,367 \\
\hline
\end{tabular}

${ }^{1}$ Pré-Inicial e Inicial: vit. A 2750,000 UI/g, vit. D3 550,000 UI/g, vit. K3 419,900 mg/kg, vit. B1 380,158 mg/kg, vit. B2 1250,00 $\mathrm{mg} / \mathrm{kg}$, vit. B6 600,188 mg/kg, vit. B12 4000,000 mcg/kg, vit. E 8750,000 UI, pantotenato de cálcio $3000.021 \mathrm{mg} / \mathrm{kg}$, niacina $8750,175 \mathrm{mg} / \mathrm{kg}$, ácido fólico $175,00 \mathrm{mg} / \mathrm{kg}$, biotina $17,000 \mathrm{mg} / \mathrm{kg}$, colina $65000,000 \mathrm{mg} / \mathrm{kg}$, metionina $419999,700 \mathrm{mg} / \mathrm{kg}$, zinco $12500,000 \mathrm{mg} / \mathrm{kg}$, ferro $12500,000 \mathrm{mg} / \mathrm{kg}$, cobre $3000,000 \mathrm{mg} / \mathrm{kg}$, manganês $15000,000 \mathrm{mg} / \mathrm{kg}$, iodo $250,067 \mathrm{mg} / \mathrm{kg}$, selênio $93,750 \mathrm{mg} / \mathrm{kg}$, cobalto $50,000 \mathrm{mg} / \mathrm{kg}$, antioxidante $1000,000 \mathrm{mg} / \mathrm{kg}$, coccidiostático $125000,000 \mathrm{mg} / \mathrm{kg}$, promot. cresc. gran negativo 6250,000 mg/kg, promot. cresc. gran positivo $125000,000 \mathrm{mg} / \mathrm{kg}$. Crescimento: vit. A 2250,000 UI/g, vit. D3 450,000 $\mathrm{UI} / \mathrm{g}$, vit. K3 417,950 mg/kg, vit. B1 300,125 mg/kg, vit. B2 1000,00 mg/kg, vit. B6 450,038 mg/kg, vit. B12 3000,000 mcg/kg, vit. E 7000,000 UI, pantotenato de cálcio 2500,083 mg/kg, niacina 7000,058 mg/kg, ácido fólico 140,000 mg/kg, biotina 14,000 $\mathrm{mg} / \mathrm{kg}$, colina $55000,000 \mathrm{mg} / \mathrm{kg}$, metionina $379999,531 \mathrm{mg} / \mathrm{kg}$, zinco 12500,000 mg/kg, ferro 12500,000 mg/kg, cobre 3000,000 $\mathrm{mg} / \mathrm{kg}$, manganês $14999,996 \mathrm{mg} / \mathrm{kg}$, iodo $250,000 \mathrm{mg} / \mathrm{kg}$, selênio $75,000 \mathrm{mg} / \mathrm{kg}$, cobalto 50,000 mg/kg, antioxidante $1000,000 \mathrm{mg} /$ $\mathrm{kg}$, coccidiostático $125000,000 \mathrm{mg} / \mathrm{kg}$, promot. cresc. gran negativo $6250,000 \mathrm{mg} / \mathrm{kg}$, promot. cresc. gran positivo $125000,000 \mathrm{mg} /$ kg. Terminação: vit. A 1000,000 UI/g, vit. D3 200,000 UI/g, vit. K3 180,050 mg/kg, vit. B1 149,858 mg/kg, vit. B2 500,000 mg/ $\mathrm{kg}$, vit. B6 249,975 mg/kg, vit. B12 1250,000 mcg/kg, vit. E 3500,000 UI, pantotenato de cálcio $1200,167 \mathrm{mg} / \mathrm{kg}, \mathrm{niacina} 70,000$ $\mathrm{mg} / \mathrm{kg}$, ácido fólico $70,000 \mathrm{mg} / \mathrm{kg}$, biotina $7,000 \mathrm{mg} / \mathrm{kg}$, colina 45000,000 mg/kg, metionina 300000,125 mg/kg, zinco 12500,000 $\mathrm{mg} / \mathrm{kg}$, ferro $12500,000 \mathrm{mg} / \mathrm{kg}$, cobre $3000,000 \mathrm{mg} / \mathrm{kg}$, manganês $15000,000 \mathrm{mg} / \mathrm{kg}$, iodo 250,067 mg/kg, selênio 56,250 mg/kg, cobalto $50,000 \mathrm{mg} / \mathrm{kg}$, antioxidante $1000,000 \mathrm{mg} / \mathrm{kg}$.

Fonte: Elaboração dos autores.

Para a determinação do desempenho das aves, os parâmetros de produção avaliados foram: ganho de peso/ave, consumo de ração/ave, conversão alimentar e viabilidade criatória, avaliados ao final de cada fase e durante todo o período experimental.

Ao final dos 42 dias, duas aves que representavam o peso médio de cada parcela experimental foram submetidas a um período de 8 horas de jejum alimentar, transportadas em veículo automotivo por um período de 30 minutos e em seguida foram imediatamente abatidas, pois segundo Oba et al. (2009), este manejo pré-abate proporciona maior incidência de problemas na qualidade da carne de frangos. 
As aves foram pesadas individualmente na plataforma de abate. Em seguida, foram insensibilizadas eletricamente através do aparelho da marca Fluxo, modelo FX 2.0, no qual as aves foram expostas por 10 segundos a $42 \mathrm{v}$ e $800 \mathrm{~Hz}$, sangradas, escaldadas, evisceradas e submetidas aos cortes comerciais para a determinação do rendimento de carcaça e cortes. Os peitos foram resfriados em solução de água + gelo e armazenados a $4{ }^{\circ} \mathrm{C}$ até a realização das análises de qualidade que foram realizadas após 24 horas.

A determinação do rendimento de carcaça foi realizada conforme metodologia descrita por Mendes (2001), onde se considerou o peso da carcaça eviscerada (sem cabeça, pés e pescoço) em relação ao peso vivo de abate. Os cortes de peito, pernas (coxa + sobrecoxa), dorso e asas tiveram seus respectivos rendimentos determinados em relação ao peso da carcaça eviscerada.

A gordura abdominal foi constituída pelo tecido adiposo presente desde a moela até o conteúdo presente ao redor da cloaca e bursa de Fabricius, conforme descrito por Smith (1993).

As análises de qualidade da carne, $\mathrm{pH}$, coloração, capacidade de retenção de água, perdas de água por cocção e força de cisalhamento foram realizadas no peito (pectoralis major) após 24 horas do abate.

$\mathrm{O}$ pH foi determinado, através da inserção de eletrodo na parte cranial do músculo pectoralis major, usando um potenciômetro (Testo 205 pHmeter). As medidas de cor foram realizadas na face ventral do filé, tomando três pontos diferentes de leitura por amostra. A medida de cor foi analisada utilizando colorímetro Komica Minolta CR10 (Osaka, Japão) e os resultados foram expressos em $\mathrm{L}^{*}$ (luminosidade), a* (componente vermelho verde) e b* (componente amarelo - azul) do sistema de cor CIELAB.

A medida de perdas de água por pressão foi realizada de acordo com o método descrito por
Barbut (1996). As perdas de água durante a cocção foram determinadas segundo Cason, Lyon e Papa (1997), em que amostras de carne do peito foram pesadas, identificadas, e embaladas em saquinhos plásticos, seladas e submetidas a cozimento em banho-maria a $85^{\circ} \mathrm{C}$ por 30 minutos. Após este procedimento as amostras foram retiradas do banho-maria, resfriadas em temperatura ambiente, desembaladas e pesadas novamente, sendo que a diferença entre o peso inicial e final das amostras correspondeu as perdas durante a cocção em relação ao peso inicial.

A força de cisalhamento foi determinada através do equipamento CT3 Texture Analyzer - Brookfield, acoplado a sonda Warner-Bratzler. Foram utilizadas as amostras de carne de peito cozidas usadas na análise de determinação das perdas por cocção, de tal forma que estas foram cortadas em tiras de $1,5 \mathrm{~cm}$ de largura, sendo colocadas com as fibras orientadas no sentido perpendicular à lâmina Warner-Bratzler, determinando-se a força máxima necessária para efetuar seu corte.

Os valores médios dos parâmetros analisados foram submetidos à análise de variância por meio do programa SAEG (Sistema para Análises Estatísticas e Genéticas versão 9.1). Foram realizadas análises de regressão para os efeitos significativos dos níveis de selênio, sendo considerado até o efeito quadrático.

\section{Resultados e Discussão}

Os resultados obtidos mostraram que o ganho de peso apresentou efeito linear negativo nos períodos de 1 a $7\left(\hat{Y}=0,12954-0,0172638 X, R^{2}=0,77\right)$ e 1 a $21 \operatorname{dias}\left(\hat{\mathrm{Y}}=0,855967-0,0846397 \mathrm{X}, \mathrm{R}^{2}=0,68\right) \mathrm{em}$ função dos níveis crescentes de selênio orgânico, mostrando assim, que até na fase inicial de criação, a suplementação de selênio orgânico causou intoxicação ou alteração no metabolismo das aves (Tabela 2). 
Tabela 2. Desempenho de frangos de corte suplementados com diferentes níveis de selênio orgânico.

\begin{tabular}{|c|c|c|c|c|c|c|c|}
\hline \multirow{2}{*}{$\begin{array}{l}\text { Variáveis de } \\
\text { Desempenho }\end{array}$} & \multicolumn{4}{|c|}{ Níveis de $\mathrm{Se} \mathrm{mg} / \mathrm{kg}$ de ração } & \multirow{2}{*}{$\begin{array}{l}\text { Efeito da } \\
\text { Regressão }\end{array}$} & \multirow{2}{*}{$\mathrm{CV} \%$} & \multirow{2}{*}{$\mathrm{R}^{2}$} \\
\hline & 0 & 0,2 & 0,4 & 0,6 & & & \\
\hline \multicolumn{8}{|c|}{$1-7$ dias } \\
\hline${ }^{1} \mathrm{GP}(\mathrm{kg})$ & 0,131 & 0,122 & 0,124 & 0,120 & Lin* & 7,017 & 0,770 \\
\hline CR (kg) & 0,160 & 0,153 & 0,153 & 0,152 & NS & 5,281 & NS \\
\hline $\mathrm{CA}$ & 1,224 & 1,252 & 1,237 & 1,274 & NS & 6,990 & NS \\
\hline VC (\%) & 100,00 & 100,00 & 98,670 & 98,660 & NS & 2,000 & NS \\
\hline \multicolumn{8}{|c|}{$1-21$ dias } \\
\hline${ }^{2} \mathrm{GP}(\mathrm{kg})$ & 0,868 & 0,818 & 0,815 & 0,817 & Lin* & 4,630 & 0,680 \\
\hline CR (kg) & 1,208 & 1,145 & 1,140 & 1,153 & NS & 4,480 & NS \\
\hline $\mathrm{CA}$ & 1,393 & 1,400 & 1,402 & 1,413 & NS & 4,133 & NS \\
\hline${ }^{3} \mathrm{VC}(\%)$ & 100,00 & 100,00 & 98,670 & 97,330 & Lin* & 2,243 & 0,890 \\
\hline \multicolumn{8}{|c|}{$1-35$ dias } \\
\hline GP (kg) & 2,150 & 2,031 & 2,104 & 2,071 & NS & 2,850 & $\mathrm{NS}$ \\
\hline CR (kg) & 3,232 & 3,099 & 3,304 & 3,185 & NS & 4,261 & NS \\
\hline $\mathrm{CA}$ & 1,503 & 1,526 & 1,570 & 1,540 & NS & 3,563 & NS \\
\hline${ }^{4} \mathrm{VC}(\%)$ & 100,00 & 100,00 & 98,670 & 97,330 & Lin* & 2,240 & 0,890 \\
\hline \multicolumn{8}{|c|}{$1-42$ dias } \\
\hline GP (kg) & 2,591 & 2,550 & 2,523 & 2,546 & NS & 3,366 & NS \\
\hline CR (kg) & 4,272 & 4,131 & 4,294 & 4,192 & NS & 3,865 & NS \\
\hline $\mathrm{CA}$ & 1,650 & 1,610 & 1,700 & 1,648 & NS & 3,782 & NS \\
\hline $\mathrm{VC}(\%)$ & 99,050 & 100,00 & 97,330 & 96,000 & NS & 0,211 & NS \\
\hline
\end{tabular}

$\mathrm{GP}=$ Ganho de peso; $\mathrm{CR}=$ Consumo de ração; $\mathrm{CA}=$ Conversão alimentar; $\mathrm{VC}=$ Viabilidade criatória; $\mathrm{CV} \%=\mathrm{Coeficiente} \mathrm{de}$ variação; * $\mathrm{P}<0,05$; NS= Não significativo $\mathrm{P}>0,05 \%$; Lin= Efeito linear;

${ }^{1} \hat{\mathrm{Y}}=0,12954-0,0172638 \mathrm{X},\left(\mathrm{R}^{2}=0,77\right) ;{ }^{2} \hat{\mathrm{Y}}=0,855967-0,0846397 \mathrm{X},\left(\mathrm{R}^{2}=0,68\right) ;{ }^{3} \hat{\mathrm{Y}}=100,313-0,447917 \mathrm{X},\left(\mathrm{R}^{2}=0,89\right) ;{ }^{4} \hat{\mathrm{Y}}=$ $100,313-0,447917 \mathrm{X},\left(\mathrm{R}^{2}=0,89\right)$.

Fonte: Elaboração dos autores.

O mesmo ocorreu para o parâmetro viabilidade criatória, nos períodos de 1 a 21 ( $\hat{Y}=100,313$ $\left.0,447917 \mathrm{X}, \mathrm{R}^{2}=0,89\right)$ e 1 a $35 \operatorname{dias}(\hat{\mathrm{Y}}=100,313$ - 0,447917X, $\mathrm{R}^{2}=0,89$ ), onde a suplementação de maiores níveis de selênio orgânico, causaram maior mortalidade (2,67\%). Jianhua, Ohtsuka e Hayashi (2000), ao avaliarem a influência da suplementação de selênio inorgânico nos níveis de 0,0;0,1; 0,3 e 0,5 $\mathrm{mg} \mathrm{Se} / \mathrm{kg}$ de ração sobre o crescimento de frangos de corte, observaram aumento nos níveis sanguíneos do hormônio T3 e da enzima iodotironina desiodase em função do aumento da concentração de selênio presente na dieta dos animais.

Os hormônios T3 e T4 regulam a atividade do metabolismo animal e afetam a taxa funcional de muitos outros sistemas do corpo. Em excesso, estimulam o metabolismo e exacerbam os efeitos do sistema nervoso simpático, causando aceleração de vários sistemas corporais. Para manter o metabolismo elevado, o animal desvia energia que antes seria destinada à produção, e dessa forma acaba ganhando menos peso, pois o gasto com a energia de mantença é maior. Além disso, animais metabolicamente estressados estão mais suscetíveis à falência dos órgãos, acarretando piora na viabilidade criatória conforme afirmam Olkowski e Classen (1995).

Os resultados encontrados discordam de Funari Júnior et al. (2010), que trabalhando com suplementação de 0,15 e $0,45 \mathrm{mg} \mathrm{Se} / \mathrm{kg}$ de ração, tanto na forma orgânica, como inorgânica para frangos de corte, observaram que a fonte não influenciou o ganho de peso, porém observaram efeito dos níveis sobre os parâmetros de desempenho, 
sendo que a suplementação de $0,45 \mathrm{mg} \mathrm{Se} / \mathrm{kg}$ de ração, proporcionou maior peso médio no período de 1 a 42 dias de criação.

O consumo de ração e a conversão alimentar, não foram afetados $(\mathrm{P}>0,05)$ pelos tratamentos experimentais em nenhum dos períodos avaliados. Estes resultados corroboram com os de Moreira et al. (2001), que ao suplementarem diferentes níveis de selênio inorgânico e orgânico na dieta de frangos de corte, não observaram efeito significativo dos tratamentos sobre os mesmos parâmetros de desempenho.

Os resultados obtidos podem ser justificados devido à participação do selênio sobre os hormônios produzidos pela tireóide. Segundo Meyer, Wagnere e Maia (2007), o mineral está relacionado com as iodotironinas desiodases que formam uma família de selenoenzimas com propriedades catalíticas distintas que ativam os hormônios tireoidianos via desiodação do anel fenólico ou tirosínico da molécula do 3,5,3',5'- tetraiodo-Ltiroxina (T4), convertendo-o para sua forma mais ativa, o 3,5,3'-triiodo-L-tironina (T3).

Os resultados referentes aos rendimentos de cortes (Tabela 3) mostram que para o rendimento de pernas houve efeito quadrático $(\mathrm{P}<0,05),(\hat{\mathrm{Y}}=$ 20,6633- 3,98603X + 7,23406 $\left.\mathrm{X}^{2} ; \mathrm{R}^{2}=0,99\right)$, sendo que esse parâmetro sofreu redução até o nível calculado de 0,2755 mg Se/kg ração.

Tabela 3. Rendimento de carcaça e partes de frangos de corte suplementados com diferentes níveis de selênio orgânico abatidos aos 42 dias de idade.

\begin{tabular}{|c|c|c|c|c|c|c|}
\hline \multirow{2}{*}{ Rendimento (\%) } & \multicolumn{4}{|c|}{ Se (mg/kg de ração) } & \multirow{2}{*}{$\begin{array}{l}\text { Efeito da } \\
\text { Regressão }\end{array}$} & \multirow{2}{*}{$\mathrm{CV} \%$} \\
\hline & 0 & 0,2 & 0,4 & 0,6 & & \\
\hline Carcaça & 75,90 & 75,94 & 76,10 & 77,21 & NS & 3,51 \\
\hline Peito & 29,52 & 30,69 & 30,00 & 30,77 & NS & 4,65 \\
\hline${ }^{1}$ Pernas & 20,68 & 20,11 & 20,28 & 20,86 & Quad* & 4,16 \\
\hline Asas & 8,08 & 7,97 & 8,21 & 9,37 & NS & 21,57 \\
\hline Dorso & 15,96 & 15,72 & 16,22 & 15,60 & NS & 8,23 \\
\hline${ }^{2}$ Gordura Abdominal & 1,68 & 1,46 & 1,41 & 1,18 & Lin* & 37,15 \\
\hline
\end{tabular}

$\mathrm{CV} \%=$ Coeficiente de variação; * $=\mathrm{P}<0,05 ; \mathrm{NS}=$ Não significativo $\mathrm{P}>0,05$; Lin= Efeito linear; $\mathrm{Quad}=$ Efeito quadrático; ${ }^{1} \hat{\mathrm{Y}}=$ 20,6633- 3,98603X + 7,23406X2 (R2=0,99); ${ }^{2} \hat{Y}=1,66808-0,786104 X,\left(R^{2}=0,92\right)$.

Fonte: Elaboração dos autores.

Resultados similares foram observados por Edens (1996) e Edens, Gowdy e Sefton (2003), que relataram redução no rendimento de pernas em função da suplementação de Se orgânico na dieta de frangos de corte. Assis et al. (2008) também observaram que a suplementação das dietas com diferentes níveis de selênio orgânico promoveu efeito quadrático em função dos tratamentos, sendo que o nível de $0,37 \mathrm{mg} \mathrm{Se} / \mathrm{kg}$ de ração, o que proporcionou o menor rendimento.

Já o rendimento de gordura abdominal, foi influenciado positivamente pelos tratamentos
$(\mathrm{P}<0,05)$, sendo observado um efeito linear descrito pela equação $\hat{Y}=1,66808-0,786104 X,\left(R^{2}=0,92\right)$. Os resultados mostram que quanto maior o nível de selênio orgânico presente na dieta, menor o rendimento de gordura abdominal em frangos de corte abatidos aos 42 dias. Este resultado discorda de Hada (2008), que ao suplementar a dieta de frangos de corte com $0,3 \mathrm{mg} \mathrm{Se} / \mathrm{kg}$, observou maior deposição de gordura abdominal nas aves.

Acredita-se que esse parâmetro foi favorecido pelos níveis de selênio presentes nas dietas, do mesmo modo que o ganho de peso. Uma vez que o 
metabolismo desses animais é acelerado, devido à maior produção dos hormônios T3 e T4, favorecida pela maior concentração de selênio. Desta forma, ocorre maior gasto energético, reduzindo assim, o acúmulo de gordura abdominal.

Os demais parâmetros de rendimento carcaça, peito, asas e dorso, não foram afetados $(\mathrm{P}>0,05)$ pela suplementação de selênio orgânico.

Com relação aos parâmetros avaliados para qualidade da carne (Tabela 4), o pH mensurado no músculo pectoralis major dos frangos, sofreu efeito
$(\mathrm{P}<0,01)$ linear crescente, isto é, quanto maior a suplementação de selênio, maior o $\mathrm{pH}$ da carne, segundo a equação $\hat{Y}=5,74974+0,284495 X\left(R^{2}=\right.$ 0,83). É possível que a influência do selênio sobre a produção dos hormônios tireoidianos responsáveis pela taxa do metabolismo afete o acúmulo de glicogênio no músculo, uma vez que quanto maior o gasto energético, menor a quantidade de glicogênio presente no músculo, que é segundo Swatland (1995), a maior reserva energética desse tecido.

Tabela 4. Resultados de pH, perda de água por cozimento (PAC), perda de água por pressão (PAP), força de cisalhamento (FC) e coloração da carne de frangos de corte suplementados com dietas contendo diferentes níveis de selênio orgânico e abatidos aos 42 dias de idade.

\begin{tabular}{|c|c|c|c|c|c|c|}
\hline \multirow{2}{*}{ Variáveis } & \multicolumn{4}{|c|}{ Se (mg/kg de ração) } & \multirow{2}{*}{ Efeito da Regressão } & \multirow{2}{*}{$\mathrm{CV} \%$} \\
\hline & 0 & 0,2 & 0,4 & 0,6 & & \\
\hline${ }^{1} \mathrm{pH}$ & 5,73 & 5,85 & 5,83 & 5,92 & Lin** & 1,55 \\
\hline PAC (\%) & 24,74 & 23,28 & 28,41 & 24,09 & NS & 12,02 \\
\hline${ }^{2} \mathrm{PAP}(\%)$ & 30,43 & 29,21 & 27,85 & 27,79 & Lin* & 10,32 \\
\hline${ }^{3} \mathrm{FC}\left(\mathrm{kgf} / \mathrm{cm}^{2}\right)$ & 3,19 & 3,59 & 2,66 & 2,79 & Lin** & 37,45 \\
\hline${ }^{4} \mathrm{~L}^{*}$ & 51,67 & 49,59 & 50,78 & 53,47 & Quad** & 5,50 \\
\hline$a^{*}$ & 1,57 & 0,73 & 0,87 & 0,70 & NS & 119,70 \\
\hline $\mathrm{b}^{*}$ & 13,78 & 12,45 & 12,78 & 12,85 & NS & 13,98 \\
\hline
\end{tabular}

$\mathrm{L}=$ luminosidade; $\mathrm{a}^{*}=$ intensidade de vermelho; $\mathrm{b}^{*}=$ intensidade de amarelo;

$\mathrm{CV} \%=$ Coeficiente de variação; $*=\mathrm{P}<0,05 ; * *=\mathrm{P}<0,01 ; \mathrm{NS}=$ Não significativo; Lin= Efeito linear; Quad = Efeito quadrático; Equações: ' $\hat{\mathrm{Y}}=5,74974+0,284495 \mathrm{X}\left(\mathrm{R}^{2}=0,83\right) ;{ }^{2} \hat{\mathrm{Y}}=30,2624-4,75046 \mathrm{X},\left(\mathrm{R}^{2}=0,92\right) ;{ }^{3} \hat{\mathrm{Y}}=3,33433-0,964443 \mathrm{X}\left(\mathrm{R}^{2}=0,41\right) ;{ }^{4} \hat{\mathrm{Y}}=$ $51,6045-14,7386 \mathrm{X}+29,9964 \mathrm{X}^{2}\left(\mathrm{R}^{2}=0,98\right)$;

Fonte: Elaboração dos autores.

Segundo Offer e Knight (1988), o pH influencia diretamente as perdas de água na carne do peito. A depleção das reservas de glicogênio no músculo faz com que não haja acúmulo de ácido láctico no músculo post mortem. Dessa forma, o $\mathrm{pH}$ final da carne permanecerá elevado, ficando bastante acima do ponto isoelétrico das principais proteínas musculares, e nessas condições, a capacidade dessas proteínas em reter água será alta (DRANSFIELD; SOSNICKI, 1999) reduzindo as perdas de água durante o processamento. Este fato foi constatado neste experimento, visto que a perda de água por pressão sofreu efeito linear negativo $(\hat{\mathrm{Y}}=30,2624$
- 4,75046X, $\mathrm{R}^{2}=0,92$ ) em função dos níveis crescentes de Se orgânico $(\mathrm{P}<0,05)$, que causaram aumento do $\mathrm{pH}$ da carne e consequentemente menor a perda de água por pressão. O resultado corrobora com os encontrados por Perić et al. (2009), que observaram que a suplementação de Se orgânico proporcionou redução das perdas de água da carne do peito.

Brossi et al. (2009) afirmam que a suculência da carne pode afetar diretamente a força de cisalhamento, pois segundo Offer e Knight (1988) e Anadón (2002), quanto maior a quantidade de água 
fixada no músculo, maior a maciez da carne. De fato isto pode ser comprovado neste experimento, visto que a força de cisalhamento sofreu efeito linear decrescente, ( $\left.\hat{Y}=3,33433-0,964443 \mathrm{X} ; \mathrm{R}^{2}=0,41\right)$, isto é, quanto menor a perda de água da carne, mais macia ela ficou.

Os valores de $\mathrm{L}^{*}$ apresentaram resposta quadrática $(\mathrm{P}<0,01)$ segundo a equação $\hat{\mathrm{Y}}=51,6045$ - 14,7386X+29,9964X $\mathrm{X}^{2}\left(\mathrm{R}^{2}=0,98\right)$, em função dos níveis crescentes de selênio orgânico presentes nas rações experimentais, enquanto que os valores de $\mathrm{a}^{*}$ e b*, e perdas por cocção não foram influenciados significativamente. Apesar da diferença na intensidade da luminosidade das carnes, estas apresentaram valores considerados normais.

\section{Conclusão}

A suplementação da ração com selênio orgânico não influenciou o desempenho das aves no período total de criação e também proporcionou melhorias ao rendimento de gordura abdominal e favoreceu parâmetros importantes relacionados a qualidade da carne.

\section{Observação}

$\mathrm{O}$ projeto foi previamente submetido à apreciação e aprovação pelo Comitê de Ética em Experimentação Animal da Universidade Estadual de Londrina (CEEA/UEL), sob número 90/09.

\section{Agradecimentos}

À Fundação Araucária pelo financiamento do Projeto e ao CNPQ pela bolsa de mestrado concedida à autora principal.

\section{Referências}

ANADÓN, H. L. S. Biological, nutritional and processing factors affecting breast meat quality of broilers. 2002. Tese (Doctor of Philosophy in Animal and Poultry Sciences) - Faculty of Virginia Polytechnic Institute and State University, Blacksburg.

ARTHUR, J. R.; NICOL, F.; HUTCHINSON, A. R.; BECKETT, G. J. The effects of selenium depletion and repletion on the metabolism of thyroid hormones in the rat. Journal of Inorganic Biochemistry, Columbia, v. 39, n. 2, p. 101-108, 1990.

ASSIS, A. P.; SOUZA, M. G.; OLIVEIRA, W. P.; BALBINO, E. M.; MARTINS, M. S.; SOARES, F. P.; OLIVEIRA, R. F. M.; DONZELE, J. L. Níveis de selênio orgânico na ração de frangos de corte mantidos em ambiente de alta temperatura. In: ZOOTEC, 2008, João Pessoa. Anais... João Pessoa: UFPB/ABZ, 2008.

BARBUT, S. Estimates and detection of the PSE problem in young turkey breast meat. Canadian Journal of Animal Science, Sherbrooke, v. 76, n. 3, p. 455-457, 1996.

BROSSI, C.; CONTRERAS-CASTILLO, C. J.; AMAZONAS, E. A.; AMAZONAS, E. A.; MENTEN, J. F. M. Estresse térmico durante o pré-abate em frangos de corte. Ciência Rural, Santa Maria, v. 39, n. 4, p. 12961305, 2009.

CANTOR, A. H.; PESCATORE, A. J.; STRAW, M. L.; FORD, M. J.; DUNLAP, M. K. Tissue selenium concentrations of broilers fed diets suplemented with selenized yeast and sodium selenite. Poultry Science, Champaigh, v. 76, p. 58, 1997. Suplemento 1.

CASON, J. A.; LYON, C. E.; PAPA, C. M. Effect of muscle opposition during rigor on development of broiler breast meat tenderness. Poultry Science, Champaigh, v. 76, n. 5, p. 725-787, 1997.

CLOSE, W. H. The role of trace mineral proteinates in pig nutrition. In: BIOTECHNOLOGY IN THE FOOD INDUSTRY, 14., 1998, Nottingham. Anais... Nottingham: Alltech's Annual Symposium, 1998. p. 469-76.

DAHLKE, F.; GONZALES, E.; FURLAN, R.; GADELHA, A. C.; MAIORKA, A.; ALMEIDA, J. G. Suplementação dietética de selênio para frangos de corte e seus efeitos sobre o empenamento. Archives of Veterinary Science, Curitiba, v. 10, n. 1, p. 27-33, 2005.

DRANSFIELD, E.; SOSNICKI, A. A. Relationship between muscle growth and poultry meat quality. Poultry Science, Champaigh, v. 78, n. 5, p.7 43-746, 1999.

EDENS, F. W. Sodium selenite versus selenium yeast in diets fed broilers: effects on performance, feathering, meat quality and yields. In: ANNUAL SYMPOSIUM OF BIOTECHNOLOGY IN THE FEED AND FOOD INDUSTRIES, 12., 1996, Kentuky. Anais... Kentuky: Nottingham University Press. Alltech's Annual Symposium, 1996. Pôster. 
EDENS, F. W.; GOWDY, K. M.; SEFTON, A.E. Resultados de campo obtidos com frangos de corte suplementados com selênio (Sel-Plex). In: EM CONTATO COM A NATUREZA. APLICAÇÕES PRÁTICAS DE TECNOLOGIAS NATURAIS, 2003, 13., Curitiba. Anais... Curitiba: Ronda Latino Americana da Alltech, 2003. p. 11-16.

FUNARI JÚNIOR., P.; ALBUQUERQUE, R.; ALVES, F. R.; MURAROLLI, V. D. A.; TRINDADE NETO, M. A.; SILVA, E. M. Diferentes fontes e níveis de selênio sobre o desempenho de frangos de corte. Brazilian Journal Veterinary Research Animal Science, São Paulo, v. 47 , n. 5 , p. $380-384,2010$.

HADA, F. H. Envolvimento da proteína, carboidrato, lipídio e selênio sobre as alterações metabólicas e bioquímicas em frangos submetidos ao calor. 2008. Dissertação (Mestrado em Zootecnia) - Universidade Estadual Paulista, Faculdade de Ciências Agrárias e Veterinárias, Jaboticabal.

JIANHUA, H.; OHTSUKA, A.; HAYASHI, K. Selenium influences growth via thyroid hormone status in broiler chickens. British Poultry Science, London, v. 84, n. 5, p. 727-732, 2000.

MEYER, E. L. S.; WAGNER, M. S.; MAIA, A. L. Expressão das iodotironinas desiodases nas neoplasias tireoidianas. Arquivo Brasileiro de Endocrinologia e Metabologia, São Paulo, v. 51, n. 5, p. 690-700, 2007.

MOREIRA, J.; SANTOS, C. D.; ABREU, C. M. P.; BERTECHINI, A. G.; OLIVEIRA, D. F.; CARDOSO, M. G. Efeito de fontes e níveis de selênio na atividade enzimática da glutationa peroxidase e no desempenho de frangos de corte. Ciência e Agrotecnologia, Lavras, v. 25, n. 3, p. 661-666, 2001.
OBA, A.; ALMEIDA, M.; PINHEIRO, J. W.; IDA, E. I.; MARCHI, D. F.; SOARES, A. L.; SHIMOKOMAKI, M. Management of transport and lairage conditions on broiler chicken breast meat qualities and DOA (Death On Arrival). Brazilian Archives Biology and Technology, Curitiba, v. 52, p. 205-211, 2009. Especial.

OFFER, G.; KNIGHT, P. The structural basis of water-holding in meat. Part 1: general principles and water uptake in meat processing. In: LAWRIE, R. A. Developments in meat science. London: Elsevier, 1988. p. 63-171.

OLKOWSKI, A. A.; ClASSEN, H. L. Sudden death syndrome in broiler chickens: a review. Poultry Avian Biological Review, Ithaca, v. 6, n. 2, p. 95-105, 1995.

PERIĆ, L.; MILOŠEVIĆ, N.; ŽIKIĆ, D.; KANAČKI, Z.; DŽINIĆ, N.; NOLLET, L.; SPRING, P. Effect of selenium sources on performance and meat characteristics of broiler chickens. Journal Applied Poultry Research, Champaigh, v. 18, n. 3, p. 403-409, 2009.

ROSTAGNO, H. S.; ALBINO, L. F. T.; DONZELE, J. L.; GOMES, P. C.; OLIVEIRA, R. F. de; LOPES, D. C.; FERREIRA, A.S; BARRETO, S. L. de T. Tabelas brasileiras para aves e suínos: composição de alimentos e exigências nutricionais. 3. ed. Viçosa, MG: UFV, 2011. $252 \mathrm{p}$.

SMITH, M. O. Parts yield of broilers reared under cycling high temperatures. Poultry Science, Champaigh, v. 72, n. 6, p. 1146-1150, 1993.

SWATLAND, H. J. On line evaluation of meat. Lancaster: Technomic, 1995. $343 \mathrm{p}$.

VIARO, R. S.; VIARO, M. S.; FLECK, J. Importância bioquímica do selênio para no organismo humano. Disciplinarum scientia série: Ciência Biológica e da Saúde, Santa Maria, v. 2, n. 1, p. 17-21, 2001. 
